\title{
Subclassification and Detection of New Markers for the Discrimination of Primary Liver Tumors by Gene Expression Analysis Using Oligonucleotide Arrays
}

\author{
Holger G. Hass ${ }^{1}$, Ulrich Vogel ${ }^{2}$, Michael Scheurlen ${ }^{3}$, and Jürgen Jobst ${ }^{4}$ \\ ${ }^{1}$ Department of Internal Medicine, Oncology and Rehabilitation, Paracelsus Hospital, Scheidegg, ${ }^{2}$ Department of Pathology, University of \\ Tübingen, Tübingen, ${ }^{3}$ Department of Gastroenterology, Oncology, Rheumatology, University of Würzburg, Würzburg, and ${ }^{4}$ Matrigene GmbH \\ Company, Reutlingen, Germany
}

Background/Aims: The failure to correctly differentiate between intrahepatic cholangiocarcinoma (CC) and hepatocellular carcinoma (HCC) is a significant clinical problem, particularly in terms of the different treatment goals for both cancers. In this study a specific gene expression profile to discriminate these two subgroups of liver cancer was established and potential diagnostic markers for clinical use were analyzed. Methods: To evaluate the gene expression profiles of HCC and intrahepatic CC, Oligonucleotide arrays (AffymetrixU133A) were used. Overexpressed genes were checked for their potential use as new markers for discrimination and their expression values were validated by reverse transcription polymerase chain reaction and immunohistochemistry analyses. Results: 695 genes/expressed sequence tags (ESTs) in HCC (245 up-/450 down-regulated) and 552 genes/ ESTs in CC (221 up-/331 down-regulated) were significantly dysregulated ( $p<0.05$, fold change $>2, \geq 70 \%$ ). Using a supervised learning method, and one-way analysis of variance a specific 270-gene expression profile that enabled rapid, reproducible differentiation between both tumors and nonmalignant liver tissues was established. A panel of 12 genes (e.g., HSP90 $\beta$, ERG1, GPC3, TKT, ACLY, and NME1 for HCC; SPT2, T4S3, CNX43, TTD1, HBD01 for CC) were detected and partly described for the first time as potential discrimination markers. Conclusions: A specific gene expression profile for discrimination of primary liver cancer was identified and potential marker genes with feasible clinical impact were described. (Gut Liver 2018;12:306-315)

Key Words: Cholangiocarcinoma; Carcinoma, hepatocellular; Oligonucleotide arrays; Gene expression analysis; Immunohistochemistry

\section{INTRODUCTION}

Worldwide, primary liver cancer is one of the most human cancer leading to an overall incidence of over 5\% of all cancer types. ${ }^{1}$ Beside hepatocellular carcinoma (HCC), cholangiocarcinoma (CC) is the second most frequent primary malignant liver tumor. Both, HCC and CC, shows an increasing incidence ${ }^{2,3}$ and especially a high incidence in Africa and Asia. Despite the high incidence of primary liver cancer in developing countries, in Europe and the USA chronic hepatitis $\mathrm{C}$ an obesity leading to chronic inflammation of the liver nonalcoholic steatohepatitis are the main reasons for an increase of patients with HCC in the next decades. ${ }^{4}$ Whereas $70 \%$ to $80 \%$ of HCC occurs in cirrhotic liver, the etiology of CC remains in many cases unclear. As for HCC liver cirrhosis reasonable to chronic viral hepatitis leads to the increasing incidence of CC in Western countries, other typical risk factor in endemic areas includes diseases leading to chronic inflammation as liver fluke infection, hepatolithiasis or anatomical abnormalities of the biliary tract (e.g., Caroli syndrome).

A correct differentiation between $\mathrm{CC}$ and $\mathrm{HCC}$ is a relative frequent problem in clinical routine and very crucial because of different treatment goals and options for both cancers. Whereas orthotopic liver transplantation is a potentially curative option for some patients with HCC, intrahepatic CC (iCC) is considered a contraindication for transplantation because of high recurrence rate and poor overall outcome. ${ }^{5}$ Actually, sorafenib and intra-arterial chemoembolization are the best treatment options for unresectable HCC, whereas chemotherapeutic agents are not beneficial in comparison to $\mathrm{CC}$, were unresectable iCCs are treated with gemcitabine- or platine-based regimen.,

As noninvasive diagnostic of HCC so-called 4-phase multi-

Correspondence to: Holger G. Hass

Department of Internal Medicine, Oncology and Rehabilitation, Paracelsus Hospital, Kurstrasse 5, Scheidegg 88175, Germany

Tel: +49-8381-501255, Fax: +49-8381-501-290, E-mail: dr.holger.hass@paracelsus-kliniken.de

Received on June 28, 2017. Revised on August 9, 2017. Accepted on August 14, 2017. Published online December 26,2017 pISSN 1976-2283 eISSN 2005-1212 https://doi.org/10.5009/gnl17277

(c) This is an Open Access article distributed under the terms of the Creative Commons Attribution Non-Commercial License (http://creativecommons.org/licenses/by-nc/4.0) which permits unrestricted non-commercial use, distribution, and reproduction in any medium, provided the original work is properly cited. 
detector computed tomography or dynamic contrast enhanced magnetic resonance imaging (MRI) are actually recommended by the American Association for the Study of Liver Disease (AASLD) and by the European Organisation for Research and Treatment of Cancer (EORTC). ${ }^{8,9}$ However, especially small and well-differentiated iCC in cirrhotic liver tissues could show an atypical contrast enhancement pattern mimicking HCC. ${ }^{10-12}$ Because of the critical importance of a correct classification of primary liver tumors a routine histological evaluation using immunohistochemistry (IHC) is indispensable ${ }^{13,14}$ in most cases. Today immunohistochemical staining against hepatocyte paraffin antigen-1 (HepPar-1), polyclonal carcinoembryonic antigen (CEA), cluster of differentiation 10 and 34 (CD10/34), $\alpha$-fetoprotein (AFP) and glypican-3 (GPC3) are used to establish the diagnosis of HCC. However, similar to noninvasive diagnostic, there are some pitfalls in clinical routine and the use of must of these markers is limited by low sensitivity leading to difficulties by the interpretation of the marker profile, ${ }^{15,16}$ especially in poorly differentiated HCC. ${ }^{17,18}$ Similar problems of low sensitivity and specificity were seen by the use of the serum markers AFP or carbohydrate antigen 19-9 (CA19-9) for discrimination of primary or secondary liver tumors. ${ }^{19,20}$

A promising approach for better distinction between HCC and iCC is the analysis of differently expressed genes in tumor tissue by gene expression analysis or real-time quantitative reverse transcription polymerase chain reaction (RT-PCR). ${ }^{21-23}$ In this study we used oligonucleotide arrays for distinction and detection of potential biomarkers of primary liver tumors.

\section{MATERIALS AND METHODS}

\section{Acquisition of samples}

Surgical tumor probes (both tumorous and corresponding nonmalignant liver tissue) were received from 29 patients with proved primary liver tumors (10 CC, 19 HCC, for more details see Table 1). Ethics committee approval was obtained by the local ethic board of the University of Tübingen (IRB No. FS 1482001) and in accordance with the Declaration of Helsinki. Only after obtained written informed consent from the patients parts of the tumor samples were used for gene expression analyses.

\section{Histopathological and preoperative noninvasive evaluation}

For each tissue sample, we obtained clinical, virological and pathological reports (including tumor typing, staging based on Union internationale contre le cancer [UICC] criteria, grading) were obtained for each tissue sample. A hematoxylin-eosin staining and additional immunohistochemical stainings with a panel of antibodies were performed routinely (HepPar-1, AFP, cytokeratin 7 [CK7], CA19-9, CD34, and CEA) for subclassification between HCC and CC and to exclude other types of malignancies.

In all patients a preoperative, noninvasive diagnostic using computed tomography (CT) and/or MRI was performed (Table 1) and these results compared with the histopathological reports.

\section{Preparation of labeled CRNA and hybridization to oligo- nucleotide arrays}

The SuperScript Choice system (Invitrogen, Carlsbad, CA, USA) was used for transcription of total RNA into doublestranded cDNA. To assembly first-strand cDNA synthesis a T7(dT24) oligonucleotide primer with a RNA polymerase promoter site was used. In vitro transcription followed after second-strand synthesis in the presence of biotin-11-cytidine triphosphate and biotin-16-uridine triphosphate (Enzo Diagnostics, New York, NY, USA). Next, biotin-labelled cRNA were fragmented $(20 \mu \mathrm{g}$ at $94^{\circ} \mathrm{C}$ for 35 minutes) and added to a hybridization solution to a final cRNA concentration $(0.05 \mathrm{mg} / \mathrm{mL})$. The solution was incubated for hybridization with an oligonucleotide array (AffymetrixGeneChip [Hu133A]), containing 22,283 probe sets for known genes or expressed sequence tags (ESTs). After staining with streptavidin-phycoerythrin a Gene Array scanner G2500A (Hewlett Packard, Palo Alto, CA, USA) was used for scanning of the hybridisation products according to the recommendations of the manufacturer.

\section{Data mining, statistical analysis, tumor subclassification and identification of altered metabolic pathways involved in human hepato- and cholangiocarcinogenesis}

The utilization of the scanned expression data was performed using the Affymetrix microarray suite (MAS version 5.0.1). For statistical analysis and post-processing of the obtained data the software programs GeneSpring version 6.1 (Silicon Genetics, Redwood City, CA, USA) and GeneExplore version 1.1 (Applied Maths, Sint-Martens-Latem, Belgium) were used. Only Chip results of different scaling factors ( 0.5 to 1.8 ) were selected for further analyses. Using the signal log ratio which is determined by the comparison of two-array results between tumorous and nontumorous tissues expression values were obtained and then $\log 2$-transformed. A p-value of less than 0.05 (t-test) and a fold-change of $\geq 2$ in $60 \%$ or more of all analyzed tumor probes were considered as significant. Using this data a specific gene expression profile was established by two-dimensional cluster and one-way analysis of variance (ANOVA) analysis.

For identification of involved specific signaling or metabolic pathways in human hepato- or cholangiocarcinogenesis, data of significantly expressed genes were transferred in GenMapp, a public domain software program 2.0 beta $^{(}$(Gladstone Institutes, San Francisco, CA, USA). GenMapp allows the graphic representation of various pathways and the expression levels of involved genes.

The statistical analyses and presentation of the data were performed in accordance with the Minimum Information About a Microarray Experiment (MIAME) criteria and will be published online at http://www.paracelsus-kliniken.de/scheidegg/Forsc- 
Table 1. Patient Demographics, Histopathological Data and Noninvasive Diagnostic Results ( $\mathrm{n}=29)$

\begin{tabular}{|c|c|c|c|c|c|c|c|}
\hline No. & Sex & Age, yr & Histology & Staging & Grading & Etiology & Noninvasive diagnostic result \\
\hline 1 & $\mathrm{~F}$ & 57 & $\mathrm{CC}$ & pT3 & G3 & HBV, LC & CT (sd: CC, metastasis) \\
\hline 2 & $\mathrm{~F}$ & 65 & $\mathrm{CC}$ & pT3 & G2 & $\mathrm{HCV}, \mathrm{LC}$ & CT/MRI (sd: CC) \\
\hline 3 & $\mathrm{~F}$ & 68 & $\mathrm{CC}$ & pT2 & G2 & $\mathrm{C} 2, \mathrm{LC}$ & CT/MRI (sd: CC/HCC) \\
\hline 4 & M & 74 & $\mathrm{CC}$ & pT3 & G2 & LC & CT/MRI (sd: CC) \\
\hline 5 & $\mathrm{~F}$ & 62 & $\mathrm{CC}$ & pT3 & G2 & PBC, LC & CT (sd: CC) \\
\hline 6 & $\mathrm{~F}$ & 47 & $\mathrm{CC}$ & pT3 & G2 & nk & MRI (sd: metastasis) \\
\hline 7 & M & 73 & $\mathrm{CC}$ & pT3 & G2 & nk & CT (sd: CC/HCC) \\
\hline 8 & $\mathrm{~F}$ & 71 & $\mathrm{CC}$ & pT2 & G2 & nk & CT/MRI (sd: CC) \\
\hline 9 & M & 52 & $\mathrm{CC}$ & pT3 & G2 & nk & CT/MRI (sd: CC, metastasis) \\
\hline 10 & M & 65 & $\mathrm{CC}$ & pT3 & G1 & nk & CT/MRI (sd: metastasis) \\
\hline 11 & M & 62 & HCC & pT4 & G3 & $\mathrm{HCV}, \mathrm{LC}$ & CT/MRI (sd: HCC) \\
\hline 12 & M & 60 & HCC & pT3 & G2 & $\mathrm{HCV}, \mathrm{LC}$ & CT/MRI (sd: HCC/CC) \\
\hline 13 & M & 75 & HCC & pT3 & G3 & $\mathrm{HCV}, \mathrm{LC}$ & MRI (sd: HCC) \\
\hline 14 & $\mathrm{~F}$ & 58 & HCC & pT4 & G3 & $\mathrm{HCV}, \mathrm{LC}$ & CT/MRI (sd: HCC) \\
\hline 15 & M & 69 & HCC & pT3 & G2 & $\mathrm{HBV}, \mathrm{LC}$ & CT/MRI (sd: CC) \\
\hline 16 & $\mathrm{~F}$ & 55 & HCC & pT3 & G3 & $\mathrm{HBV}, \mathrm{LC}$ & CT/MRI (sd: HCC, CC) \\
\hline 17 & $\mathrm{~F}$ & 78 & HCC & pT3 & G3 & HBV, LC & CT/MRI (sd: HCC) \\
\hline 18 & M & 59 & HCC & pT3 & G2 & HBV, LC & CT (sd: HCC) \\
\hline 19 & M & 60 & HCC & pT4 & G2 & $\mathrm{C} 2, \mathrm{LC}$ & CT/MRI (sd: HCC) \\
\hline 20 & M & 73 & HCC & pT3 & G2 & $\mathrm{C} 2, \mathrm{LC}$ & CT/MRI (sd: CC) \\
\hline 21 & $\mathrm{~F}$ & 69 & HCC & pT3 & G2 & $\mathrm{C} 2, \mathrm{LC}$ & CT/MRI (sd: HCC/CC) \\
\hline 22 & M & 70 & $\mathrm{HCC}$ & pT4 & G3 & $\mathrm{C} 2, \mathrm{LC}$ & CT/MRI (sd: HCC) \\
\hline 23 & $\mathrm{~F}$ & 63 & HCC & pT2 & G2 & $\mathrm{C} 2, \mathrm{LC}$ & MRI (sd: HCC) \\
\hline 24 & $\mathrm{~F}$ & 70 & HCC & pT3 & G3 & $\mathrm{C} 2, \mathrm{LC}$ & CT/MRI (sd: HCC) \\
\hline 25 & M & 54 & $\mathrm{HCC} / \mathrm{CC}$ & pT4 & G3 & $\mathrm{C} 2, \mathrm{LC}$ & CT/MRT (sd: HCC) \\
\hline 26 & $\mathrm{~F}$ & 56 & HCC & pT2 & G1 & Stea, LC & CT/MRI (sd: HCC/CC) \\
\hline 27 & M & 61 & $\mathrm{HCC}$ & pT2 & G2 & Stea, LC & CT/MRI (sd: metastasis) \\
\hline 28 & $\mathrm{~F}$ & 64 & HCC & pT3 & G2 & nk & CT/MRI (sd: HCC) \\
\hline 29 & M & 57 & HCC & pT3 & G3 & Hämo & CT/MRI (sd: HCC) \\
\hline
\end{tabular}

F, female; CC, cholangiocarcinoma; HBV, chronic hepatitis B; LC, liver cirrhosis; CT, computed tomography; sd, suspected diagnosis; HCV, chronic hepatitis C; MRI, magnetic resonance imaging; C2, alcohol abuse; M, male; PBC, primary biliary cirrhosis; nk, not known; HCC, hepatocellular carcinoma; Stea, steatohepatitis.

hung/gene-profilesHCCvs.CCC.2017.

\section{Validation of expression data by RT-PCR and IHC}

Four of the most significantly overexpressed genes in at least $80 \%$ of the HCC and CC tissue samples were used for validation of the expression levels estimated by microarray analysis: karyopherin alpha 2 (KNPA2, GPC3), serine protease inhibitor type Kazal 1 (SPINK1) and osteopontin $(O P N)$. Primers corresponding to the coding regions of these genes were designed using OLIGO primer analysis software (Molecular Biology Insights, Colorado Springs, CO, USA) and used for RT-PCR analyses using the LightCycler ${ }^{\odot}$ system (Roche Diagnostics, Mannheim, Germany). Primer sequences were as follows:

\section{OPN: 696U; GGACAGCCGTGGGAAGG}

\author{
OPN: 810L; TCAATCACATCGGAATGCTCA \\ SPINK1: 151U; GCCTTGGCCCTGTTGAGTCTA \\ SPINK1: 273L; CACGCATTCATTGGGATAAGTATTT \\ KPNA2: 328U; GAAAACCGCAACAACCA \\ KPNA2: 501L; GCCCAAGAAGGACACAAAT \\ GPC3: 1808U; CAGCAGGCAACTCCGAAGG \\ GPC3: 1929L; TGGGCACCAGGCAGTCAGT
}

For RT-PCR reactions LC RNA Amplification Kit SYBR Green I, (Roche Diagnostics) were used. Amplification was followed by melting-curve analysis and the values for the initial target concentration in each sample were determined using LightCycler ${ }^{\circ}$ software 3.5. For each patient the relative change in gene expression was estimated by pairwise comparison of tumor and nonmalignant liver tissue samples. 
For second validation of the measured gene expression data we compared the mRNA expression level estimated by gene expression analysis of CK7 and AFP in the resected tumors using IHC. In formalin-fixed paraffin-embedded tissue sections were incubated with a dilution (1:50) of a monoclonal mouse antihuman CK7 antibody (Clone OV-TL 12/30; Dako Deutschland $\mathrm{GmbH}$, Hamburg, Germany) and for AFP using a polyclonal mouse antihuman antibody (Cell Marque, Rocklin, CA 95677, USA) according to the procedures developed by the manufacturer. Because of the different used labeling scores in literature we used the following definition of marker positivity: (1) 1\% to 10\% CK7 or AFP positive tumor cells, + (low); (2) 11\% to 50\% CK7 or AFP positive tumor cells: ++ (mean), or (3) $>50 \%$ CK7 or AFP positive tumor cells, +++ (strong).

\section{RESULTS}

\section{Gene expression profiling of primary liver cancer}

On average, 43.3\% (CC) compared with 42.6\% (HCC) and $39.4 \%$ (corresponding normal liver tissue) of 22,283 genes and
ESTs were expressed in the analysed tissue samples.

Of the approximately 13,000 genes expressed in the HCC and adjacent nonmalignant tissue samples, approximately 1,200 genes were either up- or down-regulated significantly. All significantly dysregulated genes $(p<0.05$; fold change $\geq 2$ in at least 70\%) were used to generate a databank of 695 genes/ESTs in HCC (245 up-/ 450 down-regulated) and 552 genes/ESTs in CC (221 up-/ 331 down-regulated). Using this data set, we implemented a supervised learning method based on neuronal networking and a one-way ANOVA analysis to obtain a specific gene expression profile of 270 significantly dysregulated genes for HCC and CCC. Using this analytic approach a rapid, reproducible differentiation between these primary liver tumors and malignant versus nonmalignant liver tissues was possible in all cases with a positive and negative prediction value of $100 \%$ $(\mathrm{p}<0.0001)$ (Fig. 1).

\section{Involved signaling and metabolic pathways and dysregu- lated genes in hepato- and cholangiocarcinogenesis}

Of these 1,247 dysregulated genes/ESTs in primary liver can-

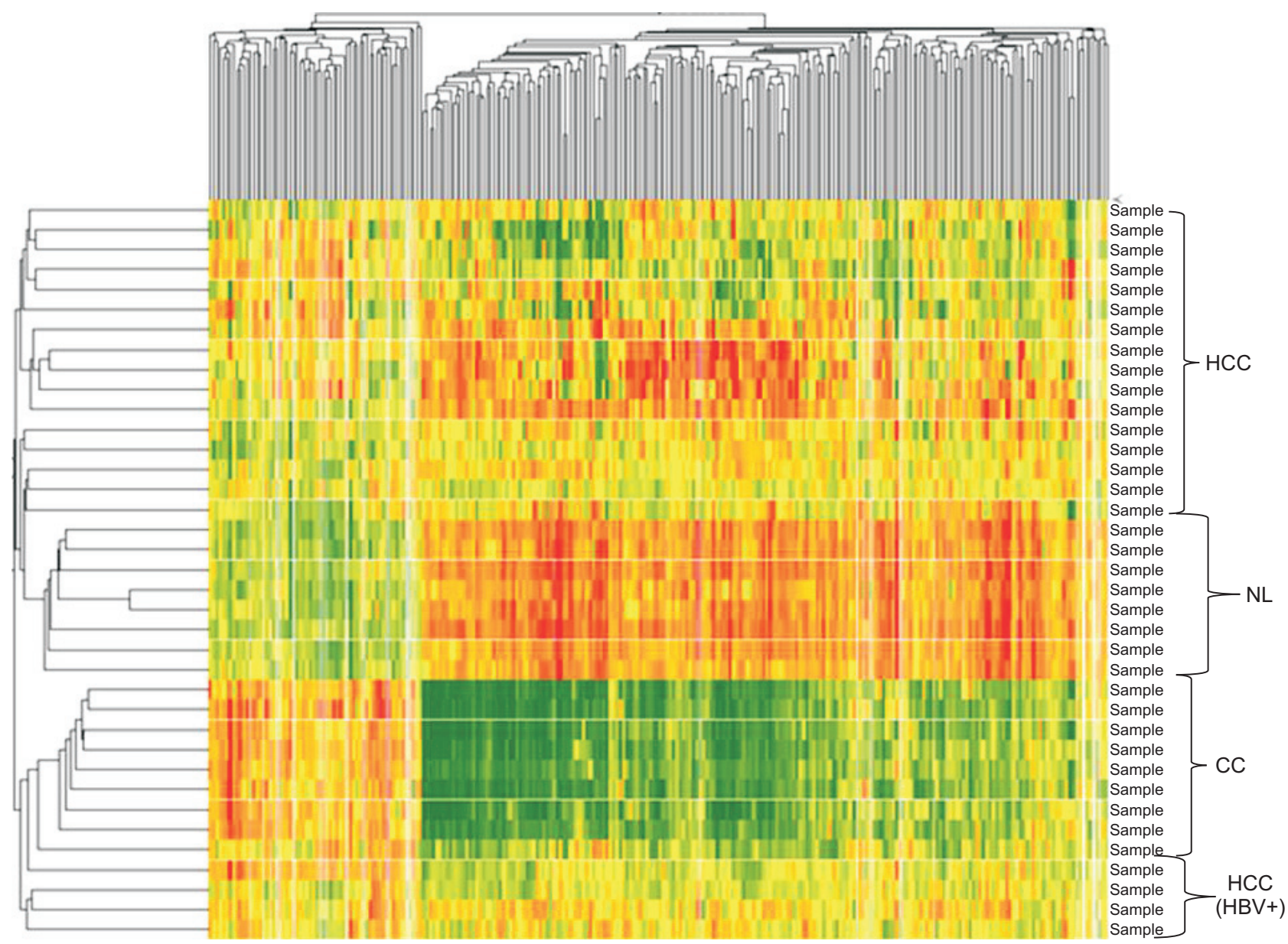

Fig. 1. Two-dimensional cluster analysis using 270 dysregulated genes in hepatocellular carcinoma (HCC) versus cholangiocarcinoma (CC) versus nonmalignant corresponding liver tissues (NL) (2-fold change in $\geq 70 \%$ : red, upregulated genes; green, downregulated genes; $p<0.05$ ). HBV, chronic hepatitis B. 
cer, 466 were upregulated and 781 genes downregulated in both tumor subgroups.

Using the GenMAPP ${ }^{\circledR}$ software involved metabolic pathways were detected and the specific gene descriptions determined by public gene databases as UniGene (U.S. National Library of Medicine, Bethesda, MD, USA) and GeneCards ${ }^{\circledR}$ (Weizmann Institute, Rehovot, Israel). By these methodical approaches 885 genes/ESTs could be dedicated to specific signaling and metabolic pathways.

Most of the significantly overexpressed genes were related to DNA replication and cell cycle regulation (HCC: 27 genes, e.g., cyclin-dependent kinase 1 [CDC2], cyclin B1 [CCNB1], tubulin gamma 1 [TUBG1], KPNA2, cell division control 4 [CDC4], cell division control 25 beta [CDC25B], calcyclin [S100A6], topoisomerase II alpha [TOP2A], proliferating cell nuclear antigen [PCNA]; CC: 15 genes, e.g., Calgizzarin [S100A11], S100A6, BUB mitotic checkpoint protein $1[B U B 1 B]$ ), genes coding for transcription factors and G-protein depending signaling (HCC: seven genes, e.g., Rac GTPase activating protein 1 [RACGAP1], Ras homolog enriched in brain [RHEB], ADP-ribosylation factor-like 2 [ARL2] and other members of the RAS oncogene family as $R A N$ and $R A B 16$; CC: six genes, e.g., sex determining region Y [SRY]-box 9 [SOX9]). Most of the other overexpressed genes could be attributed to gene groups such as genes coding for cell adhesion molecules, cytoskeleton structure, and extracellular matrix (HCC: 22 genes, e.g., OPN, SPINK1, osteonectin [SPARC], different collagens [COL1A2, COL4A2, COL6A3], capping protein [GAPG], tropomyosin 2 [TPM2], metalloproteinase 12 [MMP12], and integrin alpha 6 [ITGA6]); CC: 53 genes, e.g., OPN, ADAM metallopeptidase domain 9 [ADAM9], thymosin beta-10 [TMSB10], ITGA6, TPM2, CK 7, and CK19). Consistent with an upregulation of genes coding for cell cycle regulating proteins and DNA replication, various genes involved in ribo- somal protein synthesis and proteasome degradation were also upregulated.

In contrast, pro-apoptotic genes (HCC: four genes, e.g., clone 36 protein [CNL3], chromosome segregation 1 [yeast homolog]like [CSE1L], DNA fragmentation factor, $45 \mathrm{kD}$, $\alpha$ polypeptide [DFFA], NFKB inhibitor alpha [NFKBIA]; CC: seven genes, e.g., growth-arrest specific protein 2 [GAS2], cell death-inducing DFFA-like effector B [CIDE-B]) were downregulated in both liver tumors. Moreover, genes coding for proteins important for metabolic pathways like carbohydrate, fat and amino acid metabolism were also significant downregulated in comparison with the corresponding nontumorous liver tissue.

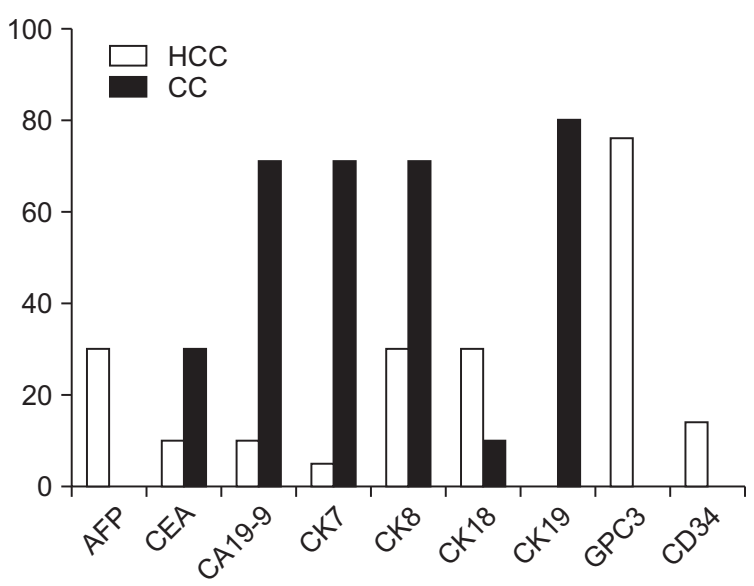

Fig. 2. Overexpressed mRNAs of established immunohistochemistry markers to discriminate primary liver cancers, as detected by microarray analysis (fold change $\geq 2$ : hepatocellular carcinoma [HCC] white; cholangiocarcinoma [CC], grey columns).

Table 2. Twelve Unique Overexpressed Genes in HCC and CC That Are Potentially Useful as Markers for the Discrimination of Primary Liver Cancers

\begin{tabular}{|c|c|c|c|c|c|c|}
\hline SwissName & Gene ID & Gene description & CC inc, $\%$ & $\mathrm{CC}$ inc, $\mathrm{fc}^{*}$ & HCC inc, $\%$ & HCC inc, $\mathrm{fc}^{*}$ \\
\hline CNX43_HUMAN & 443455 & Connexin 43 & 100 & 3.0 & & \\
\hline SPT2_HUMAN & 043291 & Serine protease inhibitor, Kunitz type, 2 & 100 & 2.5 & & \\
\hline TTD1_HUMAN & P16422 & Tumor-associated Ca signal transducer 1 & 80 & 5.9 & & \\
\hline BD01_HUMAN & Q09753 & Defensin, beta 1 & 70 & 2.7 & & \\
\hline LUM_HUMAN & P51884 & Lumican & 70 & 2.5 & & \\
\hline T4S3_HUMAN & P19075 & Transmembrane 4 superfamily member 3 & 70 & 2.2 & & \\
\hline GPC3_HUMAN & L47125 & Glypican 3 & & & 82 & 8.3 \\
\hline TKT_HUMAN & BF696840 & Transketolase & & & 82 & 3.7 \\
\hline HSP90B_HUMAN & 191175 & Heat shock protein 90 & & & 82 & 3.0 \\
\hline ACLY_HUMAN & 108728 & ATP cytrate lyase & & & 82 & 2.1 \\
\hline ERG1_HUMAN & 152427 & Squalene epoxidase & & & 82 & 2.0 \\
\hline NME1_HUMAN & 156490 & NM23A & & & 76 & 2.1 \\
\hline
\end{tabular}

HCC, hepatocellular carcinoma; CC, cholangiocarcinoma.

*Fold-change (fc) $\geq 2.0$, overexpressed in $\geq 70 \%$. 


\section{Gene expression levels of established immunohisto- chemical markers and potential new biomarkers for dif- ferentiation of primary liver cancer}

The gene expression levels of established markers used for IHC and discrimination of primary liver cancer were analyzed. Chosen marker genes for HCC included AFP, GPC3, CK8/18, and CD34 which were overexpressed in 14 (CD34) up to 76\% (GPC3) of all tumor probes. Classical marker genes for CC contained CEA, CA19-9, CK7/19. These genes showed an overexpression in 30 (CEA) up to 80\% (CK19). For a better clearance the analyzed marker genes for HCC and CC are compared in Fig. 2.

In comparison to these established markers a set of 12 genes was detected using gene expression analysis with higher expres- sion levels and better value for discrimination of primary liver cancer. For HCC six genes were chosen with significant overexpression in $76 \%$ to $82 \%$ (fold change, 2.0 to 8.3 ; downregulated in all CC). In comparison to the group of HCC 6 genes with significant overexpression (fold change, 2.2 to 5.9; downregulated in all HCC) in $80 \%$ to $100 \%$ were chosen for discrimination of CC (Table 2). A literature research (PubMed, U.S. National Library of Medicine) to investigate the potential function and importance of these genes in human carcinogenesis was performed.

\section{Validation of marker genes using RT-PCR and IHC}

To validate the expression levels of highly overexpressed candidate genes in primary liver cancer (HCC and CC), we con-
A

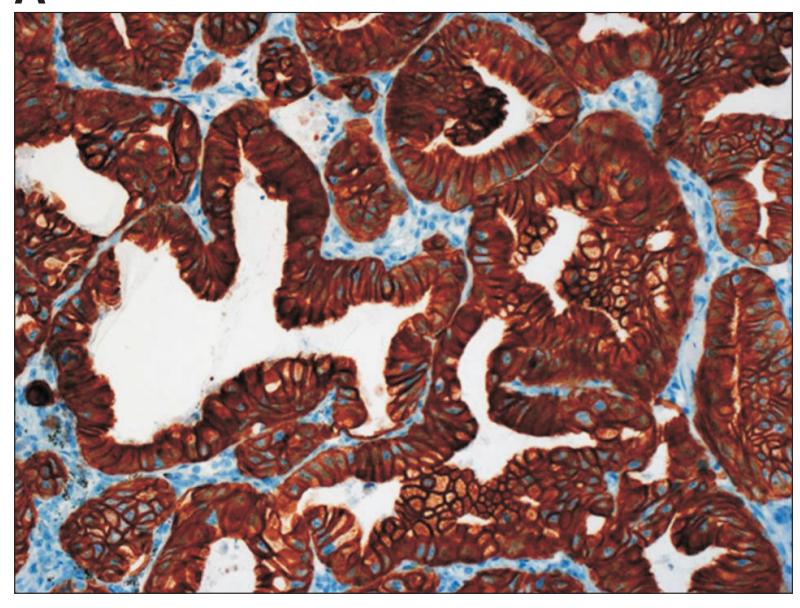

B

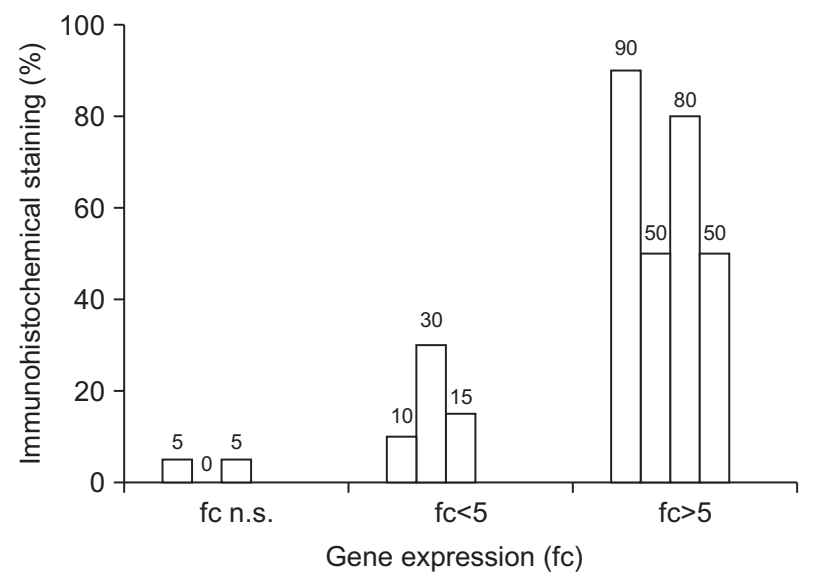

Fig. 3. (A) Immunohistochemical detection of cytokeratin 7 (CK7) in intrahepatic CC (×200). (B) Detection (\%) of CK7-positive tumor cells in correlation with the gene expression of CK7, as detected by microarray analysis (10 CC: mean expression level of CK7, fold change $=4.35$ in $70 \%$ of all CCs). $\mathrm{CC}$, cholangiocarcinoma; fc, fold change; n.s, not significant.

A

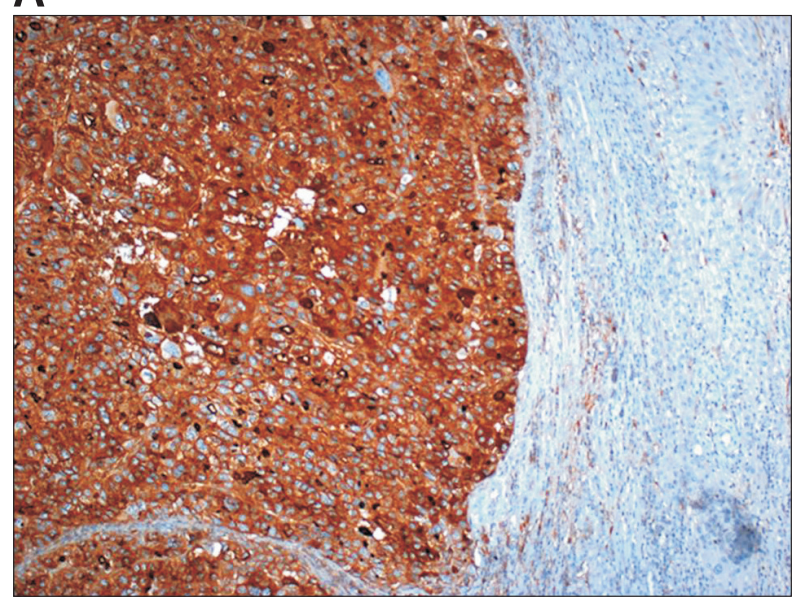

B

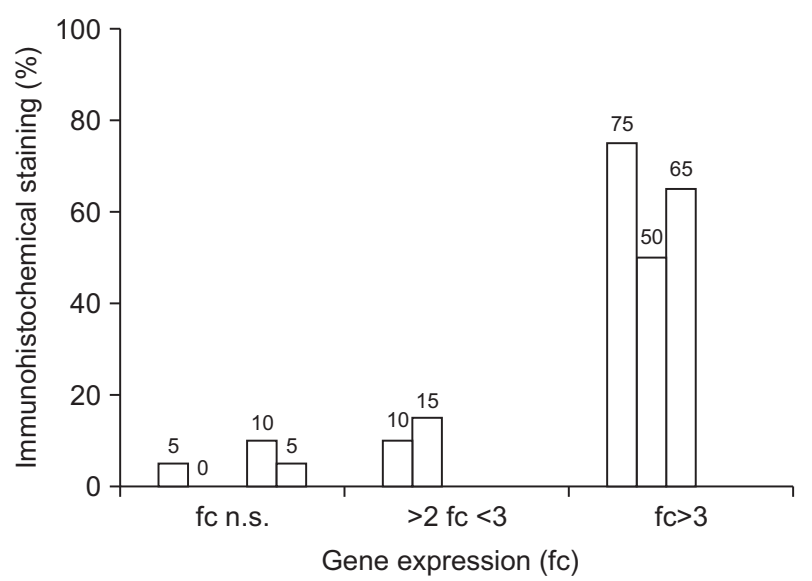

Fig. 4. (A) Immunohistochemical detection of $\alpha$-fetoprotein (AFP) in HCC ( $\times 100)$. (B) Detection (\%) of AFP-positive tumor cells in correlation with the gene expression of AFP, as detected by microarray analysis (19 HCC: mean expression level of AFP, fold change $=2.4$ in $33.3 \%$ of all HCCs). HCC, hepatocellular carcinoma; fc, fold change; n.s, not significant. 
firmed the expression levels of AFP, OPN, SPINK1, GPC3, and KNPA2 in malignant and corresponding nonmalignant tissue samples with quantitative RT-PCR. Generally, the gene expression values estimated by RT-PCR reproduced very well with the genetic levels obtained by microarray analysis. As described earlier, ${ }^{24,25}$ the dynamic reach of the gene expression levels identified by RT-PCR was significantly higher (10- to 70-fold) than the ranges estimated using the microarray technique.

In CC expression of CK7 was one of the most overexpressed genes (fold change 4.35 in $>70 \%$ of all tumor probes). Using IHC a strong correlation between gene expression levels of CK7 and CK7 positive cells was seen. In tumors with low CK7 expression only $5 \%$ of all tumor cells show a positive staining, whereas a mean expression level correlated with a stronger CK7 staining (18.3\% $\pm 9.2 \%)$. In tumors with high CK7 expression levels (fold change $>5$ ), CK7 staining was significantly stronger (67.5\% $\pm 20.6 \%, p=0.004$ ) (Fig. 3).

Similar results were seen in HCC with high AFP mRNA levels (fold change $>3$ ). In these cases AFP levels estimated by IHC were also significant overexpressed $(63.3 \% \pm 12.6 \%, p=0.021)$ in all cases (Fig. 4).

\section{DISCUSSION}

Because of the different treatment options in primary liver cancer, a fast and secure differentiation in clinical routine is indispensable for affected patients.

Despite of recent progress in noninvasive diagnostic of liver tumors using latest CT and MRI technology (e.g., quadri-phasic multidetector $\mathrm{CT}$ ) there are still some pitfalls in preoperative clarification and discrimination between HCC and iCC, especially in underlying liver cirrhosis and poorly differentiated iCCs. In our study $20 \%$ of all iCC were misdiagnosed as metastases, whereas $15.8 \%$ of all HCC were considered as iCC or metastasis in preoperative CT or MRI scans. In 21\% (HCC) and 40\% (iCC) other differential diagnoses were also postulated. As a consequence the positive predictive value ranged between $80 \%$ and $84.2 \%$.

Due to heterogeneity and similarities in morphology the histologic distinction between CC and HCC in clinical routine may also be complicated. ${ }^{13,26}$ Although several immunohistochemical markers such as cytokeratins (CK7 and CK20), and HepPar-1 are widely used to distinguish primary liver cancer, these markers can be expressed by both cancers. ${ }^{27,28}$ In addition, diagnosis of HCC can be difficult in well and poor differentiated tumors. Whereas poorly differentiated HCC or unusual subtypes as clearcell, sarcomatoid or pleomorphics variants may be mistaken for metastases, ${ }^{14,29}$ distinction of well differentiated tumor nodes from regenerative, cirrhotic liver tissue may be very difficult.

Therefore, novel diagnostic markers for a fast and secure diagnostic differentiation of primary liver tumors are required.

In the actual study we used gene expression analysis to estab- lish a unique gene expression profile for discrimination of HCC and CC. Using a primary data set of 695 genes/ESTs significantly dysregulated in HCC and 552 genes/ESTs dysregulated in CC a one-way ANOVA analysis generated a specific genetic profile of these tumors using 270 genes. This profile enables a fast and secure differentiation between all analyzed tumor probes and the corresponding, nonmalignant liver tissue in all cases with a high specify, sensitivity and positive predictive value (respectively $100 \%$ ).

In line with the clinical experience that there is no "perfect" marker for discrimination of HCC and iCC we found that the genetic expression levels of actually recommended markers for routinely application showed a wide range and often an overexpression in both liver cancers. The two markers with the highest specificity for HCC were GPC3 and AFP, both markers uniformly overexpressed in HCC (76\% and 33.3\%; 0\% CC). These data are in line with immunohistochemical data and illuminate the hitch of AFP as tumor marker in clinical routine ${ }^{30-32}$ with very low sensitivity (20\% to $60 \%$ ) and specificity (76\% to $96 \%$ ), reasonable to an overexpression and elevated serum levels of AFP in various, nonmalignant liver diseases, such as viral hepatitis or liver cirrhosis and low expression especially in small and early HCC.

Interestingly, the current recommended ${ }^{13,27}$ hepatocyte-specific marker arginase 1 (ARG1) was indeed significantly downregulated in our collective of CCs (mean fold change, -4.97 ; $80 \%$ ) but also downregulated in $68 \%$ of all HCC probes. At least on the genetic level ARG1 does not appear as "good" marker for discrimination of primary liver cancer.

For the immunohistochemical diagnosis of CC a panel of different cytokeratins (e.g., CK7 and CK19) is routinely used. In our collective these markers were significantly overexpressed in nearly complete all CC tissue (70\% to $80 \%)$ and especially the genetic expression of CK7 correlated well with the results obtained by IHC. Nevertheless, there are reports about an expression of these markers in up to $28 \%$ to $40 \%{ }^{33,34}$ also in HCC, maybe in the subgroup of tumors originating from liver stem cells. In consideration of the fact, that cytokeratins are also significantly expressed in different intestinal cancers, e.g., stomach cancer, ${ }^{35}$ the immunohistochemical discrimination between CC and secondary adenocarcinomas of the liver is often difficult in clinical routine and underlines the need for specific genetic markers for cholangiocarcinogenesis.

Adjacent to the establishment of a specific gene expression profile for subclassification of primary liver tumors another aim of the study was the detection of new and specific markers for the discrimination of these tumors in clinical routine.

By microarray analysis we found a panel of potential new marker for HCC and CC (each six genes), most of them to date not described in primary liver cancer. The most overexpressed gene in over 80\% of all HCC was the gene coding for GPC3 and distinguished the importance of this marker in clinical routine. 
Another potential marker for HCC whose was not expressed in CC is transketolase, a key protein in glucose metabolism of cancer cells. Transketolase promotes cell growth and is overexpressed in different human cancers. ${ }^{36}$ Until now there are only few reports about an upregulation of this gene in HCC. ${ }^{37,38}$ Another interesting group of genes related to hepatocarcinogenesis are different heat shock proteins (HSPs). Especially the expression of HSP90, is frequently upregulated in HCC, however, there is low evidence about the function of HSP90 in multistep hepatocarcinogenesis. One hypothesis postulates the function of this stress inducible chaperon for tumor angiogenesis and because of its strong overexpression HSP90 is current under investigations as a target for anticancer therapy. ${ }^{39}$

As specific and unique in $\mathrm{CC}$, the gene coding for the serine protease inhibitor 2, Kunitz type (SPT2) was overexpressed detected. In literature there are no reports about a dysregulation of this protease inhibitor in CC and the actual data of the protein function in human carcinogenesis is still confusing. Normally the function of this proteinase inhibitor is described as pro-apoptotic and anti-cancerous by inhibiting tumor cell invasion. ${ }^{40}$ As for SPT2 there are no data about the potential role of defensin beta in carcinogenesis of CC. Beta-defensins are especially expressed in lung epithelial cells and act as antimicrobial agents. As an important component of the innate immune response, the gene is lost at high frequencies in malignant prostatic tissue underlines the theory of the function of defensing beta as tumor suppressor ${ }^{41}$ and thereby may be indirectly elevated as a reaction of the immune system to the tumor. ${ }^{42}$ Further studies must execute to clear the function of SPT2 and defensing beta in cholangiocarcinogenesis and their utility as specific markers for CC. As for the last two reported genes, the natural role of connexin 43 (CNX43) as important gap junction protein is tumor suppressive, ${ }^{43}$ but other data suggests that connexin 43 may play a role in tumor angiogenesis and therefore in early tumor metastasis ${ }^{44}$ and serve as a potential marker of early CC.

Limitations of our study is the manageable amount of analyzed liver tumors and thereby the small group of well-differentiated tumors $(n=2)$ and the absence of rare subgroups of HCC as scirrhous carcinoma or combined hepatocellular-cholangiocarcinoma. Nevertheless, even in these cases gene expression analysis may be a helpful tool in clinical routine. ${ }^{45}$

In conclusion, a specific and unique gene expression profile which allows a fast and secure distinction of primary liver cancer was established. In comparison to noninvasive imaging and immunohistochemical analytic approaches this profile showed a higher specify and sensitivity to discriminate HCC against iCC. Second, using gene expression data potential better markers for primary liver cancer were described, in some cases for the first time in hepato- and cholangiocarcinogenesis. Further investigations must explore the use of these markers as diagnostic tools or potential molecular targets for liver cancer therapy in clinical routine.

\section{CONFLICTS OF INTEREST}

No potential conflict of interest relevant to this article was reported.

\section{ACKNOWLEDGEMENTS}

This work was supported by the fortune-program of the University of Tuebingen (No. F1281305). We thank Stephan Kaiser (MD) for his support by designing the study.

\section{REFERENCES}

1. El-Serag HB. Hepatocellular carcinoma: an epidemiologic view. J Clin Gastroenterol 2002;35(5 Suppl 2):S72-S78.

2. Davila JA, El-Serag HB. Cholangiocarcinoma: the "other" liver cancer on the rise. Am J Gastroenterol 2002;97:3199-3200.

3. McGlynn KA, Tsao L, Hsing AW, Devesa SS, Fraumeni JF Jr. International trends and patterns of primary liver cancer. Int J Cancer 2001;94:290-296.

4. El-Serag HB, Rudolph KL. Hepatocellular carcinoma: epidemiology and molecular carcinogenesis. Gastroenterology 2007;132:25572576.

5. Yao FY, Bass NM, Nikolai B, et al. A follow-up analysis of the pattern and predictors of dropout from the waiting list for liver transplantation in patients with hepatocellular carcinoma: implications for the current organ allocation policy. Liver Transpl 2003;9:684692.

6. Llovet JM, Ricci S, Mazzaferro V, et al. Sorafenib in advanced hepatocellular carcinoma. N Engl J Med 2008;359:378-390.

7. Valle JW, Wasan H, Johnson P, et al. Gemcitabine alone or in combination with cisplatin in patients with advanced or metastatic cholangiocarcinomas or other biliary tract tumours: a multicentre randomised phase II study - The UK ABC-01 Study. Br J Cancer 2009;101:621-627.

8. Bruix J, Sherman M; American Association for the Study of Liver Diseases. Management of hepatocellular carcinoma: an update. Hepatology 2011;53:1020-1022.

9. European Association for the Study of the Liver; European Organisation for Research and Treatment of Cancer. EASL-EORTC clinical practice guidelines: management of hepatocellular carcinoma. J Hepatol 2012;56:908-943.

10. Kim SA, Lee JM, Lee KB, et al. Intrahepatic mass-forming cholangiocarcinomas: enhancement patterns at multiphasic CT, with special emphasis on arterial enhancement pattern--correlation with clinicopathologic findings. Radiology 2011;260:148-157.

11. Kang Y, Lee JM, Kim SH, Han JK, Choi BI. Intrahepatic massforming cholangiocarcinoma: enhancement patterns on gadoxetic acid-enhanced MR images. Radiology 2012;264:751-760.

12. Nanashima A, Abo T, Murakami G, et al. Intrahepatic cholangio- 
carcinoma: relationship between tumor imaging enhancement by measuring attenuation and clinicopathologic characteristics. $\mathrm{Ab}-$ dom Imaging 2013;38:785-792.

13. Radwan NA, Ahmed NS. The diagnostic value of arginase-1 immunostaining in differentiating hepatocellular carcinoma from metastatic carcinoma and cholangiocarcinoma as compared to HepPar-1. Diagn Pathol 2012;7:149.

14. Lamps LW, Folpe AL. The diagnostic value of hepatocyte paraffin antibody 1 in differentiating hepatocellular neoplasms from nonhepatic tumors: a review. Adv Anat Pathol 2003;10:39-43.

15. Kakar S, Gown AM, Goodman ZD, Ferrell LD. Best practices in diagnostic immunohistochemistry: hepatocellular carcinoma versus metastatic neoplasms. Arch Pathol Lab Med 2007;131:1648-1654.

16. Chu PG, Ishizawa S, Wu E, Weiss LM. Hepatocyte antigen as a marker of hepatocellular carcinoma: an immunohistochemical comparison to carcinoembryonic antigen, CD10, and alphafetoprotein. Am J Surg Pathol 2002;26:978-988.

17. Kakar S, Muir T, Murphy LM, Lloyd RV, Burgart LJ. Immunoreactivity of Hep Par 1 in hepatic and extrahepatic tumors and its correlation with albumin in situ hybridization in hepatocellular carcinoma. Am J Clin Pathol 2003;119:361-366.

18. Minervini MI, Demetris AJ, Lee RG, Carr BI, Madariaga J, Nalesnik MA. Utilization of hepatocyte-specific antibody in the immunocytochemical evaluation of liver tumors. Mod Pathol 1997;10:686692.

19. Lok AS, Sterling RK, Everhart JE, et al. Des-gamma-carboxy prothrombin and alpha-fetoprotein as biomarkers for the early detection of hepatocellular carcinoma. Gastroenterology 2010;138:493502.

20. Marrelli D, Caruso S, Pedrazzani C, et al. CA19-9 serum levels in obstructive jaundice: clinical value in benign and malignant conditions. Am J Surg 2009;198:333-339.

21. Man XB, Tang L, Zhang BH, et al. Upregulation of glypican-3 expression in hepatocellular carcinoma but downregulation in cholangiocarcinoma indicates its differential diagnosis value in primary liver cancers. Liver Int 2005;25:962-966.

22. Wang L, Zang W, Xie D, et al. Comparison of hepatocellular carcinoma (HCC), cholangiocarcinoma (CC), and combined HCC-CC (CHC) with each other based on microarray dataset. Tumour Biol 2013;34:1679-1684.

23. Heringlake S, Hofdmann M, Fiebeler A, Manns MP, Schmiegel W, Tannapfel A. Identification and expression analysis of the aldoketoreductase1-B10 gene in primary malignant liver tumours. J Hepatol 2010;52:220-227.

24. Hass HG, Nehls 0, Jobst J, Frilling A, Vogel U, Kaiser S. Identification of osteopontin as the most consistently over-expressed gene in intrahepatic cholangiocarcinoma: detection by oligonucleotide microarray and real-time PCR analysis. World J Gastroenterol 2008;14:2501-2510.

25. Rajeevan MS, Vernon SD, Taysavang N, Unger ER. Validation of array-based gene expression profiles by real-time (kinetic) RTPCR. J Mol Diagn 2001;3:26-31.
26. Ganjei P, Nadji M, Albores-Saavedra J, Morales AR. Histologic markers in primary and metastatic tumors of the liver. Cancer 1988;62:1994-1998.

27. Fujiwara M, Kwok S, Yano H, Pai RK. Arginase-1 is a more sensitive marker of hepatic differentiation than HepPar-1 and glypican-3 in fine-needle aspiration biopsies. Cancer Cytopathol 2012;120:230-237.

28. Rullier A, Le Bail B, Fawaz R, Blanc JF, Saric J, Bioulac-Sage P. Cytokeratin 7 and 20 expression in cholangiocarcinomas varies along the biliary tract but still differs from that in colorectal carcinoma metastasis. Am J Surg Pathol 2000;24:870-876.

29. Sugiki T, Yamamoto M, Taka K, Nakano M. Specific characteristics of scirrhous hepatocellular carcinoma. Hepatogastroenterology 2009;56:1086-1089.

30. Gambarin-Gelwan M, Wolf DC, Shapiro R, Schwartz ME, Min AD. Sensitivity of commonly available screening tests in detecting hepatocellular carcinoma in cirrhotic patients undergoing liver transplantation. Am J Gastroenterol 2000;95:1535-1538.

31. Hass HG, Denzlinger C, Schäffer M, Wellhäußer U, Smith U, Markmann HU. Diagnostic and prognostic aspects of hepatocellular carcinoma: a retrospective analysis in 145 patients. J Gastroenterol Hepatol Res 2017;6:2358-2364.

32. Kokudo N, Makuuchi M. Evidence-based clinical practice guidelines for hepatocellular carcinoma in Japan: the J-HCC guidelines. J Gastroenterol 2009;44 Suppl 19:119-121.

33. Durnez A, Verslype C, Nevens F, et al. The clinicopathological and prognostic relevance of cytokeratin 7 and 19 expression in hepatocellular carcinoma: a possible progenitor cell origin. Histopathology 2006;49:138-151.

34. Uenishi T, Kubo S, Yamamoto T, et al. Cytokeratin 19 expression in hepatocellular carcinoma predicts early postoperative recurrence. Cancer Sci 2003;94:851-857.

35. Park SY, Kim HS, Hong EK, Kim WH. Expression of cytokeratins 7 and 20 in primary carcinomas of the stomach and colorectum and their value in the differential diagnosis of metastatic carcinomas to the ovary. Hum Pathol 2002;33:1078-1085.

36. Ahopelto K, Böckelman C, Hagström J, Koskensalo S, Haglund C. Transketolase-like protein 1 expression predicts poor prognosis in colorectal cancer. Cancer Biol Ther 2016;17:163-168.

37. Tan GS, Lim KH, Tan HT, et al. Novel proteomic biomarker panel for prediction of aggressive metastatic hepatocellular carcinoma relapse in surgically resectable patients. J Proteome Res 2014;13:4833-4846.

38. Corona G, De Lorenzo E, Elia C, et al. Differential proteomic analysis of hepatocellular carcinoma. Int J Oncol 2010;36:93-99.

39. Cheng W, Ainiwaer A, Xiao L, et al. Role of the novel HSP90 inhibitor AUY922 in hepatocellular carcinoma: potential for therapy. Mol Med Rep 2015;12:2451-2456.

40. Kobayashi H, Suzuki M, Tanaka Y, Kanayama N, Terao T. A Kunitz-type protease inhibitor, bikunin, inhibits ovarian cancer cell invasion by blocking the calcium-dependent transforming growth factor-beta 1 signaling cascade. J Biol Chem 2003;278:7790-7799. 
41. Bullard RS, Gibson W, Bose SK, et al. Functional analysis of the host defense peptide human beta defensin-1: new insight into its potential role in cancer. Mol Immunol 2008;45:839-848.

42. Arimura Y, Ashitani J, Yanagi S, et al. Elevated serum betadefensins concentrations in patients with lung cancer. Anticancer Res 2004;24:4051-4057.

43. Sirnes S, Bruun J, Kolberg M, et al. Connexin43 acts as a colorectal cancer tumor suppressor and predicts disease outcome. Int $\mathrm{J}$ Cancer 2012;131:570-581.
44. Elzarrad MK, Haroon A, Willecke K, Dobrowolski R, Gillespie MN, $\mathrm{Al}-\mathrm{Mehdi} \mathrm{AB}$. Connexin-43 upregulation in micrometastases and tumor vasculature and its role in tumor cell attachment to pulmonary endothelium. BMC Med 2008;6:20.

45. Xue TC, Zhang BH, Ye SL, Ren ZG. Differentially expressed gene profiles of intrahepatic cholangiocarcinoma, hepatocellular carcinoma, and combined hepatocellular-cholangiocarcinoma by integrated microarray analysis. Tumour Biol 2015;36:5891-5899. 